\title{
Extension of a New Method for Surface Reconstruction from Cross Sections
}

\author{
Joaquín Pina Amargós $^{1}$ and René Alquézar Mancho² \\ ${ }^{1}$ Centro de Estudios de Ingeniería de Sistemas (CEIS), Polytechnic Institute "José A \\ Echeverría" (CUJAE), Havana, Cuba \\ jpina@ceis.cujae.edu.cu \\ ${ }^{2}$ Departament de Llenguatges i Sistemes Informàtics (LSI), Universitat Politècnica \\ de Catalunya (UPC), Barcelona, Spain \\ alquezar@lsi.upc.es
}

\begin{abstract}
The principal steps of a new method to solve the problem of surface reconstruction from parallel cross sections are presented in this paper. This method constitutes the extension of one previously proposed by the authors using the skeleton to solve the investigation problem. The method guarantees the correct topology of the surface without altering the original contours. Some results are shown that illustrate the excellent performance of the method in particular difficult cases not solved previously. All the cases analyzed are manipulated in the same way. In real cases, the global time complexity improves the quadratic time of the quickest consulted methods.
\end{abstract}

\section{Introduction}

The problem of reconstructing the surface of a solid object from a series of parallel planar cross sections has been treated by the specialized literature in the last three decades $[3,5,8,9,14]$. A cross-section is formed by a set of closed contours defining the boundary of the material of interest to be reconstructed. As a distance separates the sections, information is often lost of the places where the ramifications occur in the surface of interest. This causes a shape difference and a different number of contours in adjacent sections (Fig. 1). A way to approach this problem is creating intermediate sections representing the place where the ramifications occur [8-10].

In this work, two verification criteria are taken into account. These criteria have been used by many authors (e.g. $[1,3,9,10,14]): 1)$ The proposed solution should obtain a topologically correct surface (in general, closed and not intercepted with itself) and 2) A resample of the same surface, in the place occupied by the original sections, should produce the original data.

The authors of the present work previously proposed a new method [10] to solve the branching problem. The method is based on the skeletonization technique to create new contours, corresponding to an artificial intermediate slice that models the level where branching occurs. This method makes a successful treatment of several ramifications cases without violating the verification criteria. However, it neither deals with the cases of local protuberances not present in the adjacent section (Fig. 1a) 
nor the cases of multiple ramification where more than a contour of a section should connect with more than a contour of the adjacent section (Fig. 1b).

Only few of the consulted works $([1,8])$ solve the "many to many" ramification case (Fig. 1b) and none has reported the solution to the case in which surface portions twist (Fig. 1c).
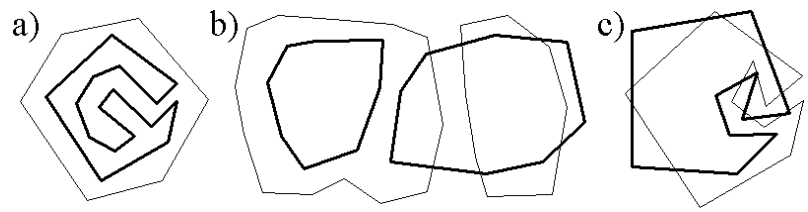

Fig. 1. Top view of several difficult cases

In this work a new method constituting an extension of [10] is proposed to offer an efficient and automatic solution to the investigation problem. The method reconstructs a topologically correct surface without modifying the data of the original sections.

Below, in Section 2, the main steps of the method are described. Their complexity is analyzed in Section 3. Finally, in Section 4, some results are shown in different complicated examples.

\section{Proposed Method}

For each original section, the initial data are a set of closed contours that define the boundary of the material of interest to be reconstructed. The proposed method consists of applying five steps to each pair of adjacent sections. The pseudocode of the main subroutine would be:

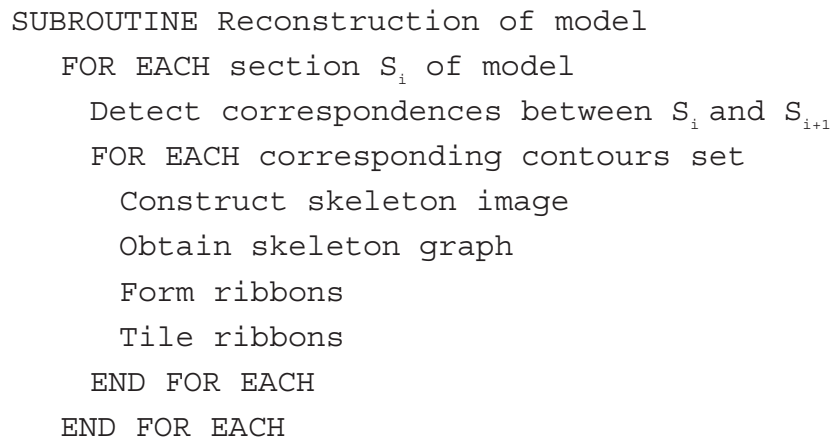

The first step determines the correspondences existing among the contours of the sections analyzed. In this work, an overlapping method was used. This method establishes that two contours should be connected by a surface if the projections of the material of interest they wrap up overlap to a certain threshold. All the projections are made on a plane parallel to the original sections (usually the $X Y$ ). The following steps are explained below and, by way of example, their results are shown in Fig. 2. 


\subsection{Skeleton Image Construction}

The second step uses the same projection information than the previous step to build an image $I$ representing the area that separates the material of interest of the analyzed contours (Fig. 2b). Then a thinning algorithm is applied, similar to the one used in [6], to obtain the skeleton $E(I)$ (shown as thick lines in Fig. 2c). Optionally, the short hair can be eliminated (Fig. 2d). For more details concerning this step, [10] should be consulted. The skeleton built in this way offers very valuable information to reconstruct in a correct and quick way the surface that connects the corresponding contours [12].

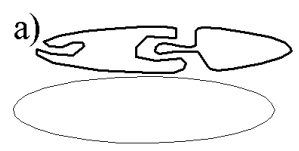

e)

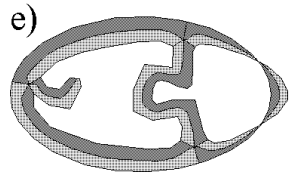

b)

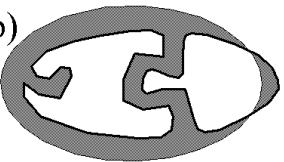

f)

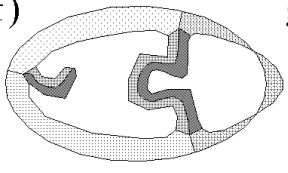

c)

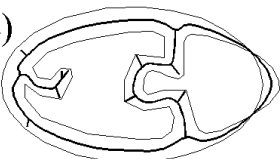

g)

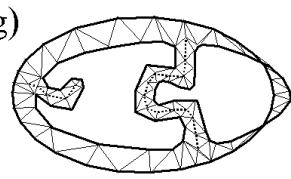

d)

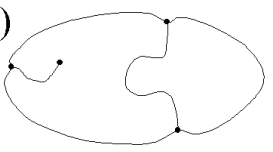

h)

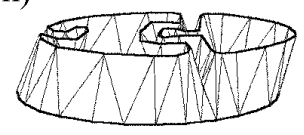

Fig. 2. Steps of the proposed method

\subsection{Skeleton Graph Obtaining}

Each black pixel of the skeleton image $E(I)$, built in the previous section, is included in the skeleton graph $G$. Structurally, $G$ is formed by a list of nodes or extreme vertices $V E$ (thick dots in Fig. 2d) and a list of arcs or rails $L$ (lines in Fig. 2d). Each node $V E$ contains its coordinates $(x, y)$ and an ordered circular list of its connections $N$. Each connection $N$ contains a rail $L$ and the pixel of $L$ to which $V E$ is connected, called neighboring vertex $V V$. For convenience, the order of the connections follows the distribution of the neighboring vertices $V V$ counterclockwise around $V E$. Each rail of $G$ contains two extreme vertices and a list of the intermediate pixels that form the rail.

\subsection{Formation and Fusion of Ribbons}

In this step, the close relationship that exists between the image and its skeleton is used to simplify the final tiling, dividing the area to be reconstructed into parts called ribbons (Fig. 2e). Each ribbon is composed by a rail $L$ of $G$ and a portion $P C$ of one of the contours analyzed. $L$ and $P C$ are near and bear similar shape so that there is no other rail or contour portion inside the ribbon conformed. The foundation of this decomposition is discussed in [12].

As a result of the previous steps, the rails $L$ and extreme vertices $V E$ of $G$ are available. To form the ribbons we need to determine the portions $P C$ of the original contours. During the reconstruction process, the endpoints of each contour portion should 
be linked so that the union of the contour portions associated with each rail of the skeleton produces the original contours (Fig. 2e). Hence, in the union, these extreme vertices of portions are the only repeated points. In this way, the surface reconstruction between two adjacent sections is reduced to the union of all the ribbon reconstruction. The pseudo-code of an algorithm that guarantees the correct and quick selection of the contour portions to form the ribbons is presented below.

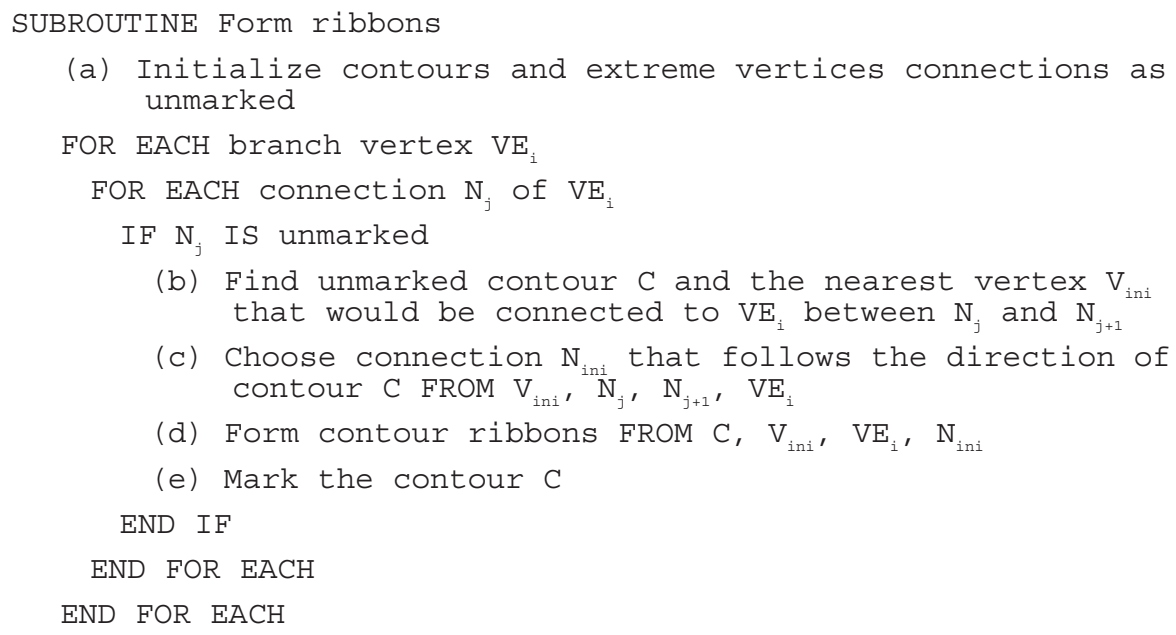

The first step (a) is responsible for labeling all contours and rails (connections between extreme vertices) as unmarked. In step (b), the vertex $V_{i n i}$ of an unmarked original contour $C$ that is met at the minimal distance from $V E$, but on the right side of the lines $\left(V E, V V_{j+1}\right),\left(V V_{j}, V E\right)$, is found (Fig. 3a). In step (c), the connection whose $V V$ is located on the same side as $V_{i n i+l}$ regarding the line $\left(V E, V_{i n i}\right)$ is selected (Fig. 3b). This ensures that, when forming the ribbons related to the contour $C$, the path followed in the rails has the same direction as $C$. In step (d), the call to the subroutine that forms the ribbons related to $C$ is made. Its pseudo-code is given next.

d)

a)
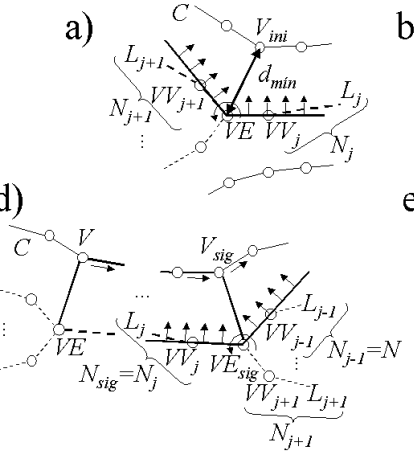

b)

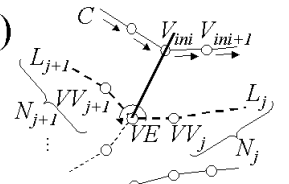

e)

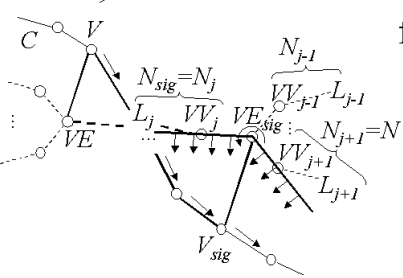

c)

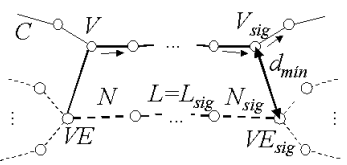

f)

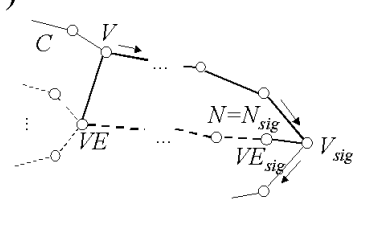

Fig. 3. Principal steps of the formation of ribbons 


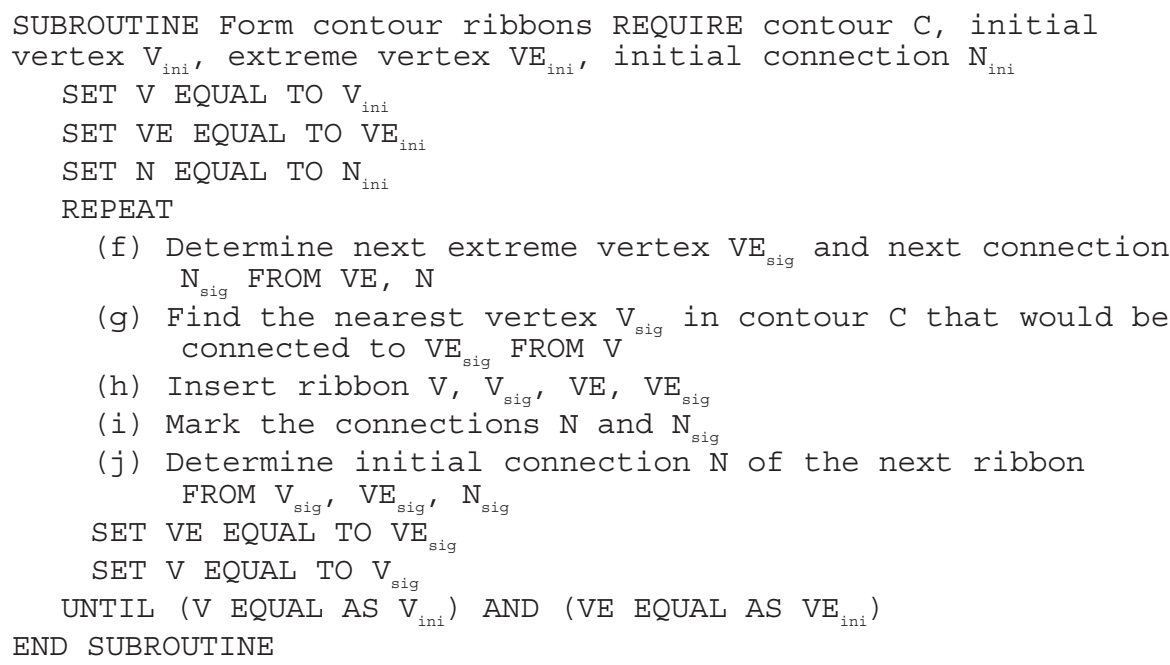

After having executed the steps (f)-(i), that are self-explained, it is necessary to determine the initial connection $N$ of the next ribbon. The three possible situations for step (j) to be executed are shown in the Fig. 3d-e-f. If $V E_{\text {sig }}$ is terminal, then $N=N_{\text {sig }}$ (Fig. 3f). If $V E_{s i g}$ is branch and $V_{\text {sig }}$ is on the right side of the straight lines $\left(V E_{s i g}, V V_{j}\right)$, $\left(V V_{j-1}, V E_{s i g}\right)$, then $N=N_{j-1}$ (Fig. 3d). Otherwise, the rail and the contour get crossed an odd number of times and then $N=N_{j+1}$ (Fig. 3e).

Optionally, to simplify the result, the adjacent ribbons whose borders belong to contours of different sections may be fused. In this way, only the skeleton vertices that are involved in ramifications remain (Fig. 2f).

\subsection{Tiling of Ribbons}

As described in [12], a ribbon is composed by a rail $L$ and a contour portion $P C$ that keep to each other proximity and shape similarity. This property can be exploited to tile the surface it forms using some simple and quick algorithm [5]. In addition, the first verification criterion mentioned in Section 1 can always be satisfied.

Finally, in the fifth step of the main procedure, the tiling of each ribbon is performed and the final surface is obtained as their union (Fig. 2g-h). The height of the skeleton vertices is intermediate to the analyzed sections, which guarantees the second verification criterion.

\section{Complexity Analysis}

The overall complexity of the proposed method is $O(n \cdot m)$, where $n$ is the number of vertices and $m$ is the number of contours in the analyzed adjacent sections.

For the calculation of the complexity of the first step, it is taken for granted that the number of pixels to process is proportional to $n$. Both the construction of the skeleton image and the extraction of its skeleton graph can be performed in linear time $O(n)$. 
The graphics of Fig. 4 show the results of the execution times of these steps in a real example composed by 151 sections, 449 contours and 91941 vertices. Their tendencies (thick lines) confirm the indicated linear time complexities.
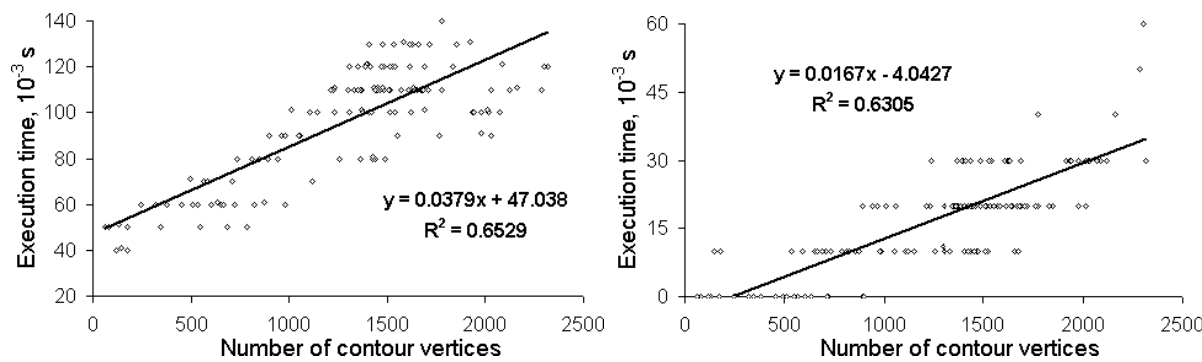

Fig. 4. Results of tests for construction of skeleton image (left) and graph (right) from different contours (Running in PC with Intel Pentium ${ }^{\circledR}$ processor at $736 \mathrm{MHz}$ and RAM of 128 Mbyte)

The complexity of the fourth step is dominated by the initial search of the contour and nearest vertex. This step is run as many times as contours there exist in the analyzed sections. As in each call to this step, the vertices belonging to the already marked contours are not treated, its complexity is $O(n \cdot m)$. This is obtained from:

$$
T=O\left(\sum_{i=0}^{m-1}\left(n-\frac{i \cdot n}{m}\right)\right) .
$$

A very quick algorithm is used for the ribbon reconstruction (fifth step), which presents a linear complexity $O(n)[5]$.

\section{Results and Discussion}

Next, some results of the application of the proposed method are shown in different synthetic examples. For more details [11] should be consulted.

In a case of two contours with very different shape, similar to Fig. 1a, the results of surface reconstruction using three classical methods (greedy, optimization and contour composition; similar to $[5,7,13]$, respectively) are shown in Fig. 5a-c, whereas the result of the proposed method is displayed in Fig. 5d. In the Figs. 5e-i, different ramification examples (without holes in the sections) are shown where a contour ramifies in two (5e-g) and three (5h-i) contours; Figs. 5g and 5i contain the results of our method. Another example refers to the existence of holes in some of the sections (Fig. 5k-1). A barely treated case in the consulted literature occurs when several contours of a section should be connected to several contours of the contiguous section; the results of our method on two such examples (the latter also with a hole) can be appreciated in the Figs. $5 \mathrm{j}, 5 \mathrm{~m}$. A case not approached by the consulted literature is shown in Fig. 6 in which a part of the surface twists abruptly between the sections. 


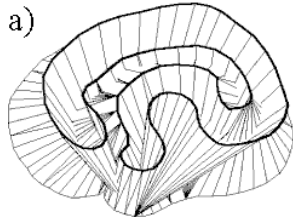

e)

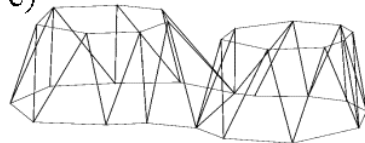

h)
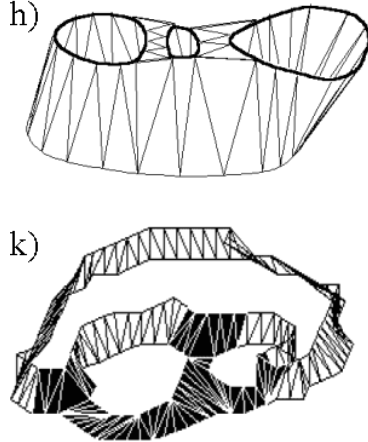

b)

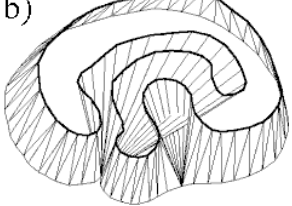

f)

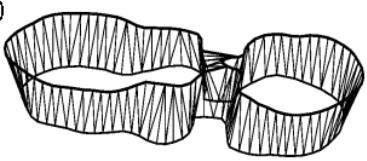

i)

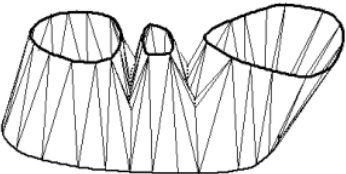

1)

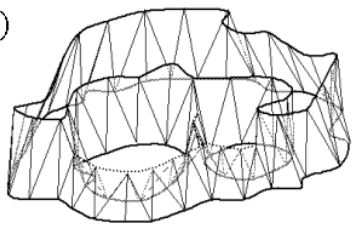

d)

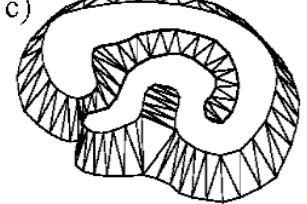

g)

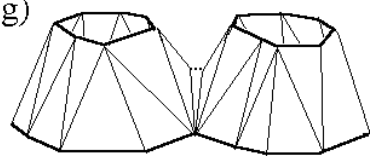

j)

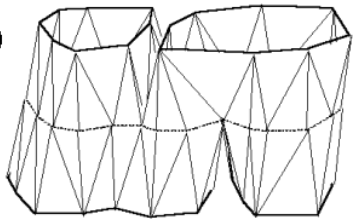

m)

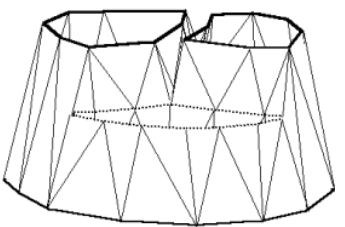

Fig. 5. 3D view of some reconstruction examples. a), b) are taken from [1]; c), f) from [13]; e) is taken from [4] and h), k) from [2]. d), g), i), j), l) and $\mathrm{m}$ ) are results of the proposed method
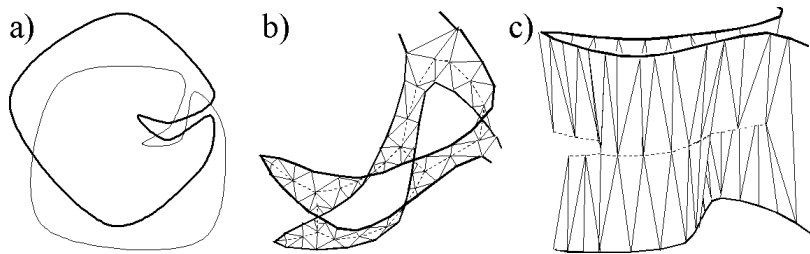

Fig. 6. Contours (a), detail of tiling $(b)$ and 3D view (c) of a very difficult example

All the analyzed cases show the quality of the results not only from the aesthetic point of view, but also in the satisfaction of the verification criteria enunciated in Section 1 . The rails related to the ramification are inserted at an intermediate height of the original sections (dotted-lines in Figs. 5, 6).

\section{Conclusions}

The main steps of a new method to reconstruct a surface from a set of cross-sections have been described. This method constitutes the extension of one previously pro- 
posed by the authors using the skeleton to solve the investigation problem [10].

The proposed method always reconstructs the surface of the whole projected area separating the material of interest between each pair of adjacent sections. It guarantees the correct topology of the reconstructed surface, because the new vertices, that model the places where the contours ramify, are inserted at an intermediate height of the adjacent sections without altering the original contours.

The method is general, simple and quick. It permits to manipulate in a same way all the cases reported in the literature and, even, one not tried by other authors. Its overall complexity is $O(n \cdot m)$, where $n$ is the number of vertices and $m$ is the number of contours in the analyzed adjacent sections. This improves the complexity $O\left(n^{2}\right)$ of the quickest consulted methods.

Some application results have been shown in different examples that, regardless of their high degree of complexity, illustrate the excellent performance of the method.

\section{References}

1. Bajaj, C.L., Coyle, E.J., Lin, K.N.: Arbitrary topology shape reconstruction from planar cross sections. Graphical Models and Image Processing. 58 (1996) 524-543

2. Barequet, G., Sharir, M.: Piecewise-linear interpolation between polygonal slices. Computer Vision and Image Understanding. 63(2) (1996) 251-272

3. Barequet, G., Shapiro, D., Tal, A.: Multilevel sensitive reconstruction of polyhedral surfaces from parallel slices. The Visual Computer. 16(2) (2000) 116-133

4. Boissonnat, J.D.: Shape reconstruction from planar cross sections. Computer Vision, Graphics and Image Processing. 44 (1988) 1-29

5. Christiansen, H.N., Sederberg, T.W.: Conversion of contour line definitions into polygonal element mosaics, Computer Graphics. 13 (1978) 187-192

6. Kégl, B., Krzyzak, A.: Piecewise linear skeletonization using principal curves. IEEE Transactions on Pattern Analysis and Machine Intelligence. 24(1) (2002) 59-74

7. Keppel, E.: Approximating complex surfaces by triangulation of contour lines. IBM Journal of Research and Development. 19 (1975) 2-11

8. Klein, R., Schilling, A., Straßen, W.: Reconstruction and simplification of surface from contours. Graphical Models. 62(6) (2000) 429-443

9. Oliva, J.M., Perrin, M., Coquillart, S.: 3D reconstruction of complex polyhedral shapes from contours using a simplified generalized Voronoi diagram. Comp. Graphics Forum, 15(3) (1996) C397-C408

10.Pina, J., Alquézar, R.: A new method to solve the branching problem in surfaces of threedimensional models reconstructed from parallel cross sections. Revista Cubana de Investigación Operacional, Universidad de La Habana, Cuba (to appear)

11.Pina, J., Alquézar, R.: Results of a new method for surface reconstruction from tomographic images. In Proc., V Cong. Soc. Cub. Bioingeniería, T0090, Havana, Cuba (2003)

12.Pina, J., Alquézar, R.: Reconstruction of surfaces from cross sections using skeleton information. In Proc. 8th Iberoamerican Congress on Pattern Recognition, Havana, Cuba (2003)

13.Sederberg, T.W., Klimaszewski, K.S., Hong, M.: Triangulation of branching contours using area minimization. Int. J. of Computational Geometry \& App., 8(4) (1998) 389-406

14.Surazhsky, T., Surazhsky, V., Barequet, G., Tal, A.: Blending polygonal shapes with different topologies. Computers \& Graphics, 25 (2001) 29-39 